\title{
Discrete Light Source Estimation from Light Probes for Photorealistic Rendering
}

\section{Farshad Einabadi}

einabadi@intel-vci.uni-saarland.de

Oliver Grau

oliver.grau@intel.com
Intel Visual Computing Institute

Saarbrücken, Germany

Intel Corporation
Applications like rendering of images using computer graphics methods are usually requiring more sophisticated light models to give better control. Complex scenes in computer generated images are requiring very differentiated light models to give a realistic rendering of the scene. That usually includes a high number of (virtual) light sources to model a scene to reproduce accurate shadows and shadings. In particular in the production of visual effects for movies and TV the real scene lighting needs to be captured very accurately to give a realistic rendering of virtual objects into that scene. In this context the light modeling is usually done manually by skilled artists in a time consuming process.

This contribution describes a new technique for estimation of discrete spot light sources. The method uses a consumer grade DSLR camera equipped with a fisheye lens to capture light probe images registered to the scene. From these probe images the geometric and radiometric properties of the dominant light sources in the scene are estimated. The first step is a robust approach to identify light sources in the light probes and to find exact positions by triangulation. Then the light direction and radiometric fall-off properties are formulated and estimated in a least square minimization approach.

There are a number of advantages in our approach. First, the probing camera is registered using a multi-camera setup which requires the minimum amendments to the studio. Second, we are not limited to any specific probing object since the properties of each light are estimated based on processing the probe images. In addition, since the probing camera can move freely in the area of interest, there are no limits in terms of the covered space. Large field of view of the fisheye lens is also beneficial in this matter.

Calibration and Registration of Cameras. We propose a two-step calibration and registration approach. In the first step, a planar asymmetric calibration pattern is used for simultaneous calibration of the intrinsics and the pose of all the witness cameras and the principal camera using a bundle adjustment module. In the next step, parameters of witness cameras are kept fixed and the probing camera is registered in the same coordinate system by using color features of an attached calibration rig.

Position Estimation. To estimate the 3D position vectors of the light sources, one needs to shoot rays from every detected light blob in all probe images and triangulate the corresponding rays from at least two probe positions for each source. Figure 1 summarizes the required steps.

\section{Inputs:}

A couple of registered light probe images

Intrinsic calibration parameters of the probing camera

\section{Output:}

3D position vectors of light sources

Steps:

Detect light blobs in all probe images

Match light blobs to their corresponding light source

For all detected light sources

Shoot rays from corresponding light blobs

Triangulate computed rays

Return the estimated 3D position of the light source

Figure 1: Pseudocode for estimating positions of the light sources

Dominant Direction and Fall-off Curve Estimation Observed intensity of a light source at each probe position is considered to be related to the brightness of its corresponding blob in the thresholded probe image. The brightness of each blob is also intuitively defined as summation over its pixel values. However, the probing device must be radiometrically calibrated to find this relation and count for all the present effects of the camera-lens system (Figure 2).

After this calibration, for a light source $j$ with $3 \mathrm{D}$ position $L_{j}$, the goal is to find its dominant direction, $\overrightarrow{l_{j}}$, and parameters of its intensity fall-off

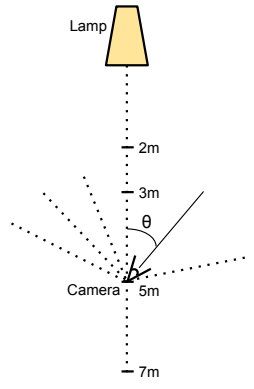

(a)

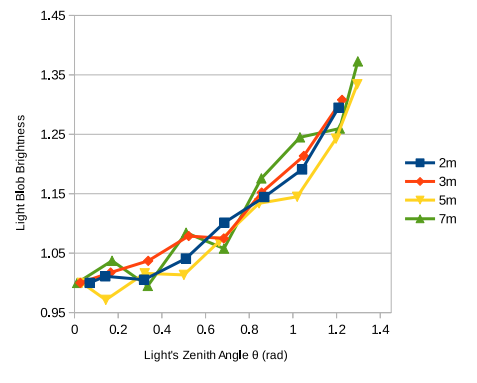

(b)
Figure 2: Radiometric calibration of the probing camera-lens system: (a) sampling process (b) distance-normalized blob brightness values w.r.t. zenith angle $\theta$ of the lamp

function, $I_{j}(\phi)$, that minimize

$$
\sum_{i=0}^{m}\left(I_{j}\left(\phi_{i j}\right)-\frac{B_{i j} \cdot\left\|P_{i}-L_{j}\right\|^{2}}{C(\theta)}\right)^{2}
$$

with $m$ the number of probes where the light source $j$ is visible in them and $P_{i}$ s their corresponding $3 \mathrm{D}$ positions. $B_{i j}$ is brightness of light blob $j$ in probe $i$ and the terms $\left\|P_{i}-L_{j}\right\|^{2}$ and $C(\theta)=\alpha \theta^{2}+1$ compensate for its quadratic attenuation w.r.t. probing distance and its brightness factor based on its place on camera's sensor plane, respectively. The angle $\phi_{i j}$ is also defined as $\phi_{i j}=\angle\left(\overrightarrow{l_{j}}, P_{i}-L_{j}\right)$.

In our formulation we assume a quadratic intensity fall-off for spot lights $I_{j}\left(\phi_{i j}\right)=k_{1} \phi_{i j}^{2}+k_{2}$ where the maximum intensity, $k_{2}$, happens at the dominant direction $\phi=0$ and $-k_{2} / k_{1}$ is the beam angle. The chosen $I_{j}$ can model the coarse fall-off characteristics of a real studio spot lamp with a limited number of probe observations.

Experiments. We verify our proposed approach on a real dataset recorded in our studio of a scene illuminated with two tungsten halogen lamps. Table 1 shows the quantitative estimation results. Figure 3 shows renders of a reference fish 3D model side by side to its real appearance at two different positions using these estimations.

\begin{tabular}{cccccc}
\hline$\#$ & & Position & Direction & Intensity & Beam Angle \\
& & $L(\mathrm{~m})$ & $\vec{l}$ & $k_{2}$ & $-k_{2} / k_{1}$ \\
\hline \multirow{4}{*}{1} & Estimated & $(2.76,3.75,1.95)$ & $(-0.8,-0.35,-0.46)$ & 2.75 & $56.22^{\circ}$ \\
& Measured & $(-,-, 1.84)$ & $(-0.80,-0.41,-0.43)$ & $4.0 e 3$ lux & $58.0^{\circ}$ \\
& Error & $(-,-, 0.11)$ & $3.86^{\circ}$ & - & $1.78^{\circ}$ \\
\hline \multirow{4}{*}{2} & Estimated & $(-1.11,1.15,1.78)$ & $(0.47,0.52,-0.71)$ & 8.98 & $50.50^{\circ}$ \\
& Measured & $(-,-, 1.73)$ & $(0.49,0.51,-0.71)$ & $1.59 e 4 \operatorname{lux}$ & $50.3^{\circ}$ \\
& Error & $(-,-, 0.04)$ & $0.88^{\circ}$ & - & $0.2^{\circ}$ \\
\hline
\end{tabular}

Table 1: Estimation results for the lamps in our dataset assuming a quadratic fall-off curve
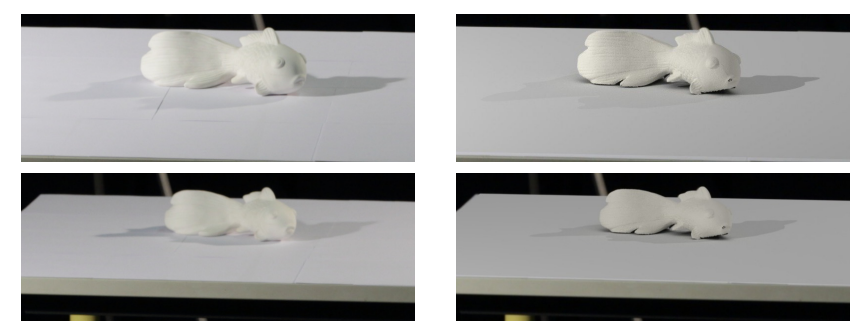

Figure 3: Visual comparison of renders of the reference object (right) to the real ones (left) 